CAmerican Psychological Association, 2021. This paper is not the copy of record and may not exactly replicate the authoritative document published in the APA journal. Please do not copy or cite without author's permission. The final article is available at: https://doi.org/10.1037/xhp0000917"

\title{
Selective Suppression of Taboo Information in Visual Word Recognition: Evidence for Cognitive Control on Semantics
}

\author{
Michele Scaltritti ${ }^{1}$, Remo Job $^{1}, \&$ Simone Sulpizio ${ }^{2}$ \\ ${ }^{1}$ Dipartimento di Psicologia e Scienze Cognitive, Università degli Studi di Trento \\ ${ }^{2}$ Dipartimento di Psicologia, Università di Milano-Bicocca
}

Word Count: 5382 (excluding title, references, author affiliations, acknowledgments, tables, figures and figure legends, appendix, and abstract)

\section{Author Note}

None of the authors has any conflict of interest to disclose.

We are Grateful to Virginia Passini for her help in running the experiment.

Correspondence concerning the article should be addressed to: Simone Sulpizio, Department of Psychology, University of Milano-Bicocca, Piazza dell'Ateneo Nuovo, 1 20126 Milano, Italy, email: simone.sulpizio@unimib.it 


\begin{abstract}
The present study investigated the role of cognitive control on semantic information during visual word recognition by exploiting taboo stimuli in a lexical decision task. We relied on delta-plots and electromyography to assess different hypothetical mechanisms of cognitive control. Previous research suggests that taboo stimuli slow down the performance across a variety of tasks, due to their attention-grabbing nature. One possibility is that cognitive control counteracts the detrimental effects of taboo connotation by actively dampening such prepotent yet task-irrelevant information. Consistent with this hypothesis, we found a reversal of taboo interference effect in slowest responses, signaling the deployment of a selective suppression mechanism that needs time to fully accrue. For electromyographic data, we focused on partial errors (trials in which a sub-threshold activation of the incorrect response hand) to index response monitoring processes intervening to prevent and correct errors. We found no modulation of the likelihood of partial errors and, more general, of response accuracy as a function of taboo connotation. Taken together, the results suggest that cognitive control may intervene to selectively suppress fast-acting and distracting taboo information, indicating a controlled semantic processing that optimizes activation to match task-relevant goals.
\end{abstract}

Keywords: visual word recognition; lexical decision; cognitive control; taboo words; semantics; partial errors

\title{
Statement of Significance
}

This study highlights the flexibility of conceptual processing during visual word recognition, by providing evidence of control mechanisms that operate at the semantic level in order to meet task-relevant goals and optimize performance. 
Orthographic processing and the mapping of orthographic input onto corresponding semantic and phonological representations are the core components of reading. However, visual word recognition also crucially hinges upon attentional factors and cognitive control. Consistently, visuospatial attention has been deemed important for word (e.g., McCann et al., 1992) and letter identification (e.g., Aschenbrenner et al., 2017). Further, executive attention seems to flexibly bias the reliance on different processing pathways and regulate information flow between representational levels (e.g., Yap et al., 2008; O’Malley \& Besner, 2008; Scaltritti et al., 2013) as a function of list composition and task demands (for reviews and discussions, see Balota \& Yap, 2006; Besner et al., 2016).

The present study investigates the role of executive attentional control in semantic processing during visual word recognition. Semantic representations are not merely retrieved from a long-term representational store, but also dynamically processed to match task- and context-relevant goals (Lambon Ralph et al., 2017). Here, we focus on how superordinate functions that support goal-directed behaviors by maintaining task-relevant information and suppressing interfering ones (e.g., Botvinick \& Braver, 2015) may be called into play with respect to semantic information activated by printed words. To reach this aim, we ran a lexical decision experiment featuring taboo and non-taboo words and pseudowords. The taboo connotation served as a proxy for a semantic content potentially requiring control, due to its detrimental influence on the achievement of task goals.

Taboo - i.e., socially inappropriate - words are typically found to require longer processing times than neutral words in line with the notion that, because of their social relevance and salience, taboo words capture participants' attention, at the expense of task performance (MacKay et al., 2004). Consistently, lexical decision experiments have reported detrimental effects of the taboo connotation on reaction times (e.g., Carretié et al., 2008; Sulpizio et al., 2019; 2020). Taboo words have also been found to yield an increased 
interference compared to non-taboo ones when used as distractors within picture-word interference (Hansen et al., 2017; Mädebach et al., 2018; White et al., 2016; 2017) and Stroop paradigms (MacKay et al., 2004; Siegrist, 1995). Taken together, these previous findings seem to point towards a pervasive influence of taboo words irrespective of their status as targets or distractors within different experimental paradigms. However, little is known about how this detrimental anchoring to the taboo information is resolved in order to accomplish the task-relevant goal. One possibility is that cognitive control intervenes to selectively suppress the interference exerted by taboo stimuli.

The notion of selective suppression has been largely investigated in the literature on cognitive control, particularly with reference to the Simon task. In this context, the interference exerted by the location dimension in incompatible trials is reduced (and shows a reversal) in slower responses (for review, see van den Wildenberg et al., 2010) where selective suppression processes have more time to be fully implemented and counteract the location information. Although the Simon effect and the interference exerted by taboo words are clearly different phenomena, in both cases cognitive control needs to face a prepotent yet task irrelevant feature of the target stimuli and its detrimental effects on performance. We hypothesize that a candidate mechanism to resolve taboo-interference during visual word recognition may be represented by a selective suppression process operating at the semantic level. Selective suppression would dampen the activation of taboo information and thus prompt a reduction, or even a reversal, of taboo interference when reaction times (RTs) are relatively long, i.e., when selective suppression has time to fully accrue.

Crucially, in our experiment, the taboo connotation was dissociated from stimulus lexicality, and thus from any response alternative. To reach this goal, we created pseudowords by changing one single letter within both taboo and non-taboo word stimuli (e.g., elifante, derived from elefante, 'elephant' vs. copezzolo, from capezzolo 'nipple') so that 
taboo connotation and lexicality were orthogonally manipulated. This choice builds on the notion that pseudowords can trigger semantic activation, as consistently suggested by recent evidence in the framework of distributional semantics. Specifically, semantic vectors for pseudowords can be computed by exploiting the vectors of the constituent letter n-grams (Hendrix \& Sun, 2020). The resulting semantic properties (e.g., semantic neighborhood density; orthography-to-semantics consistency) affect response latencies (Hendrix \& Sun, 2020; see also Cassani et al., 2020; Chuang et al., 2020), similarly to what happens with real words (e.g., Marelli \& Amenta, 2018; Amenta et al., 2017). Recent evidence has further shown that taboo pseudowords yield behavioral and electroencephalographic effects (Sulpizio et al., 2020) consistent with the notion that the taboo connotation can be activated even in this class of stimuli. In the context of our experiment, the ability of highly word-like pseudowords to trigger the activation of taboo connotation is of pivotal importance in order to address the issue of cognitive control of a prepotent semantic feature that is not confounded with task-relevant dimensions (i.e., stimulus lexicality).

Other than via the selective suppression of the interfering semantic feature, a different possibility is that cognitive control may intervene post-lexically, at the level of response monitoring. In the field of spoken word production, evidence suggests that speakers attempt to prevent any erroneous lexicalization of taboo stimuli due to their embarrassing and inappropriate connotation. The monitoring system would thus detect (and correct) errors involving taboo utterances more often than neutral ones and this increased carefulness would make the speakers slower. For example, using the picture-word interference paradigm, Dhooge and Hartsuiker (2011) showed that pictures with an unrelated taboo word distractor are named more slowly and more accurately than pictures with an unrelated non-taboo word distractor. Also, in a speeded version of the task, participants were less likely to erroneously 
name the distractor instead of the picture, when the former was a taboo word compared to a non-taboo one (for converging evidence, see also Motley et al., 1981; 1982).

Although in lexical decision no overt verbal response is required, as noted, previous studies using this task have shown that responses are slower but tend to be more accurate for taboo words compared to neutral stimuli (Sulpizio et al., 2019), potentially pointing towards an increased reliance on response monitoring for taboo stimuli. Further, even in the absence of overt verbalization, cognitive control may still operate at a peripheral level of response monitoring to prevent a potential inflation of errors triggered by the distracting nature of taboo stimuli. To investigate these monitoring-related possibilities, we recorded the electromyographic activity associated with button-press responses and focused on partial errors, i.e. trials in which the muscle associated with the incorrect response is covertly activated before the correct response is delivered (e.g., Burle et al., 2002; van den Wildenberg et al., 2010). If the system increases response monitoring processes for taboo stimuli, a higher proportion of partial errors (and an overall higher accuracy) may be expected for this category of items. In fact, the monitoring system would more promptly detect erroneous responses and correct them before they are finally issued.

In summary, we hypothesize that cognitive control may intervene to counteract the detrimental interference exerted by the taboo connotation in two different ways. On the one hand, cognitive control may selectively suppress the interfering taboo connotation, yielding a reduction of the interference effect within trials featuring slower responses, i.e., where suppression has the time to be fully implemented. On the other hand, the control system may intervene only at a later post-lexical stage, by enhancing response monitoring to prevent errors triggered by the distracting information. In turn, this may result in a higher proportion of partial EMG errors (possibly coupled with higher accuracy) for taboo stimuli, signaling the intervention of the monitoring system in correcting erroneous responses. 
In addition to the key analyses outlined above, we also performed - a posteriori - some control analyses to corroborate our interpretations. In fact, cognitive control may be flexibly enhanced as a function of the recent experience with interfering information (e.g., Gratton et al., 1992; Botvinick et al., 2001) that in our experiment should consist in taboo stimuli. Thus, for both indexes of cognitive control (taboo interference as a function of response speed and proportion of partial errors) we performed sequential analyses to capture the influence of the immediately preceding trial. Further, we analyzed how response accuracy varies as a function of response speed. Although our experiment was built to dissociate taboo connotation from lexicality, there is the chance that the early availability of such a prepotent semantic feature may have biased participants towards "word" responses, yielding an inflated chance of lexicalization errors for taboo pseudowords, particularly in fast responses (i.e., lexical capture). All these control analyses are presented in more detail in the results section.

\section{Method}

\section{Participants}

Forty students ( 35 females, mean age $=21.7, S D=2.66$ ) of the University of Trento took part in the experiment and received course credits or $5 €$ for their participation. They were all Italian native speakers and reported normal or corrected-to-normal vision and no history of neurological, learning, or motor disabilities. The study was approved by the Ethical Committee of The University of Trento and participants signed an informed consent document before the experiment.

\section{Stimuli}

Ninety taboo words (selected from ITABOO, Sulpizio et al., 2020) and 90 non-taboo words (selected from Italian adaptation of the Affective Norms for English Words, Montefinese et al., 2014) were used. Taboo words were socially-inappropriate words belonging to the domains of sexuality, insults, and scatology/disgust (e.g., erection, vagina, faggot, bastard, 
excrements, vomit). Non-taboo words were socially appropriate words belonging to different semantic domains such as objects, animals, plants, and others (e.g., helicopter, hairpin, elephant, willow, cherry). Taboo words and non-taboo words were comparable in terms of several psycholinguistic variables and differed in valence and arousal (Table 1), with Taboo words showing significantly higher arousal and lower valence ratings compared to Non-taboo stimuli.

Ninety taboo pseudowords (e.g., glante, from glande, 'glans'; sperna, from sperma, 'sperm'; beldracca, from baldracca, 'slut) and 90 non-taboo pseudowords (e.g., autanno, from autunno, 'autumn'; dettoglio, from dettaglio, 'detail'; padelta, from padella, 'pan') were created by changing one letter from the word stimuli. The first and the last letter of the word were never changed (except for one item for which the final letter was erroneously substituted). Taboo pseudowords were included in the experiment a) in order to investigate the effects of taboo content independently of the lexical status of the stimuli and b) in order not to make the taboo dimension predictive of the response. The two sets of pseudowords were comparable in terms of number of letters, orthographic neighborhood size, and orthographic Levenshtein distance. Pseudowords and words were also comparable in terms of these latter 3 variables. Psycholinguistic properties of the stimuli are listed in Table 1.

Stimuli were partitioned into 2 subsets so that a) for each participant, each stimulus appeared only once, either as a word or as a pseudoword, and b) all the stimuli (either as words or as pseudowords) appeared equally often across participants. Thus, each subset consisted of 90 words - 45 taboo and 45 non-taboo words - and 90 pseudowords - 45 taboo and 45 non-taboo pseudowords. Within each subset, taboo and non-taboo stimuli, as well as words and pseudowords, were comparable in terms of the same psycholinguistic properties reported above. The same applies for the comparison between the two subsets. Half of the participants were administered with the first subset of items, the other half with the second. 


\section{Table 1}

Psycholinguistic properties of the stimuli used in the experiment

\begin{tabular}{|c|c|c|c|c|c|c|c|c|c|}
\hline Variables & & Words & & Pset & vords & & & & \\
\hline & $\mathrm{Tb}$ & $\mathrm{NTb}$ & $p$ & $\mathrm{~Tb}$ & $\mathrm{NTb}$ & $p$ & Words & PWs & $p$ \\
\hline $\begin{array}{l}\text { Frequency } \\
(\log )\end{array}$ & 5.65 & 6.01 & $>.19$ & - & - & - & - & - & - \\
\hline N. of Letters & 7.92 & 8.04 & $>.62$ & 7.92 & 8.04 & $>.62$ & 7.98 & 7.98 & 1 \\
\hline Orth. N. & 2.66 & 2.41 & $>.62$ & 2.14 & 3.26 & $>.09$ & 2.53 & 2.70 & $>.68$ \\
\hline OLD & 2.37 & 2.25 & $>.28$ & 2.51 & 2.42 & $>.43$ & 2.31 & 2.46 & $>.05$ \\
\hline Concreteness & 6.26 & 6.31 & $>.76$ & - & - & & - & - & - \\
\hline Imageability & 6.05 & 6.35 & $>.14$ & - & - & & - & - & - \\
\hline Valence & 4 & 5.79 & $<.001$ & - & - & & & & \\
\hline Arousal & 5.1 & 4.93 & .04 & - & - & & - & - & - \\
\hline
\end{tabular}

Note. $\mathrm{N}$. of Letters = number of letters; Orth. $\mathrm{N}=$ orthographic neighborhood size; OLD = orthographic Levenshtein distance. Frequency values (log-transformed) and Orthographic Neighborhood Size were taken from the SUBTLEX-IT database (Crepaldi, Keuleers, Mandera, \& Brysbaert, 2013). Concreteness, Imageability, Valence and Arousal scores were taken from ITABOO (Sulpizio et al., 2020) and the Italian adaptation (Montefinese et al., 2014) of the Affective Norms for English Words database (ANEW; Bradley \& Lang, 1999), for taboo and non-taboo words, respectively.

\section{Apparatus and procedure}

Participants first read and signed the informed consent document, and completed a brief questionnaire collecting demographic information and others related to the state of health information. After installing the electrodes for the recording of EMG activity (see the next section), participants performed a lexical decision experiment. The experimental procedure and the acquisition of behavioral data were controlled using the E-Prime 2 software running on a laptop. Participants sat in an armchair at a distance of $\sim 40 \mathrm{~cm}$ from the computer screen, holding a joypad in their hands with their thumbs resting on the upper triggers. They were instructed to categorize letter strings as words or pseudowords performing thumb button- 
presses with the right or the left hand on the joypad trigger. Response button was counterbalanced across participants. Participants could take a break after 45 trials. A set of 8 practice trials preceded the experiment.

Each trial started with a fixation cross, presented at the center of the screen. Its duration was randomly selected between 5 possible alternatives (400, 450, 500, 550, $600 \mathrm{~ms})$ and was immediately followed by the stimulus, which remained on the screen until the participant's response or for a maximum of $1500 \mathrm{~ms}$. Trials were separated by a blank screen displayed for 700ms. All stimuli appeared in white Courier New font (18 pt) on a black background.

\section{EMG recording and processing}

EMG activity of the flexor pollicis brevis was acquired via an eego sports system (ANT Neuro, Enschede, The Netherlands), from two pairs of bipolar electrodes placed $\sim 2 \mathrm{~cm}$ apart on the thenar eminences of both hands, with a sampling rate of $1,000 \mathrm{~Hz}$.

EMG data were high-pass (10 Hz; order 2 Butterworth filters) and notch $(50 \mathrm{~Hz})$ filtered offline. The continuous signal was segmented into epochs going from -300 before stimulus onset until $1900 \mathrm{~ms}$ afterwards. The onset of EMG activity within each epoch was detected using an automated algorithm. Specifically, the root mean square of the signal was computed over a centered sliding window of $11 \mathrm{~ms}$. The resulting data were transformed into z-scores on the basis of the average and the standard deviation of the amplitude within each epoch. The onset of the EMG signal was defined as the first of a series of at least 10 consecutive samples in which $\mathrm{z}$ was above 1.

The algorithm was applied both to the signal associated with the correct and the incorrect response hands. The former marked the onset for correct responses and partialcorrect responses (i.e., trials characterized by a sub-threshold activation of the muscle associated with the correct response before the incorrect response is delivered). The second 
marked the onset of errors and partial-errors (i.e., trials characterized by a sub-threshold activation of the muscle associated with the incorrect response before the correct response is delivered). EMG onsets for full responses (i.e., correct responses and errors) and partial ones (partial errors and partial correct) were submitted to separate visual inspection procedures performed on the raw EMG signal. For correct responses and errors, epochs in which the algorithm failed to accurately detect the onset of EMG activity $(6.70 \%)$ were excluded from subsequent chronometric analyses. For partial errors and partial correct responses, the visual inspection procedure was performed to ensure the presence of a true partial response. The sensitivity of the algorithm, in fact, made it prone to false-positives, in which random fluctuations of the signal were marked as onsets of EMG activity. Epochs were classified as containing partial errors or partial correct responses only when the screening procedure ensured that a) a visually detectable sub-threshold EMG activation was present, and b) the timing of the onset was accurate. Epochs containing partial errors $(7.85 \%)$ or partial correct responses $(0.54 \%)$ were not considered in the accuracy analyses. Epochs containing multiple separate sub-threshold activations (either in one channel or in both) were excluded from chronometric analyses and were not identified as partial errors or partial correct responses. Partial correct responses were sparse, and thus not further analyzed.

\section{Statistical Analyses}

All the chronometric measures relying on the time elapsing from stimulus onset until the onset of EMG activity were analyzed via linear mixed-effects models. Measures focusing on response accuracy or on the presence vs. absence of partial errors were analyzed using generalized mixed-effects models to accommodate the binomial nature of the dependent variables. Analyses were conducted using the lme4 library (version 4_1.1-20; Bates et al., 2015) in R (R Core Team, 2015). All the models included random intercepts for participants and items. Fixed effects were assessed via likelihood ratio tests comparing models in which 
the fixed term under examination was present vs absent. Fixed terms were retained only in case their exclusion would determine a significant decrease in goodness-of-fit. In case interactions were significant, all lower-order terms were retained in the final model. To examine how the difference between taboo and non-taboo stimuli unfolds as a function of response speed (i.e., delta-plot analyses, e.g., de Jong et al., 1994), observations within each participant and experimental condition were sorted into 5 quantiles as a function of the timing of EMG onset (first quantile $=$ fastest $20 \%$ of the responses; fifth quantile $=$ slowest $20 \%$ of the responses). The resulting variable (Quantile) was treated as a fixed effect. Non-linearities were fitted using orthogonal quadratic polynomials for the fixed term corresponding to the Quantile variable. Non-linear relationships were assessed by comparison with an equal model featuring only linear terms via likelihood ratio tests.

For eight items, the proportion of correct responses across all the participants was below the estimated chance level (i.e., $<.56)$. These items were removed from all the subsequent analyses. All the analyses featuring EMG onset times were replicated using reaction times (i.e., time elapsing from the onset of the stimulus until the actual responsebutton press) and yielded the same pattern of results.

\section{Results}

\section{EMG Onset Times}

Analyses on EMG onset times revealed only the presence of a significant Lexicality effect $\left(\chi^{2}\right.$ $[1]=100.38, p<.001)$, with faster responses for words compared to pseudowords $(b=-$ 97.10, $S E=9.02, t=-10.77$ ). Neither the effect of Stimulus Category (Taboo vs Non-Taboo; $\left.\chi^{2}[1]=0.10, p=.75\right)$, nor the Lexicality X Stimulus Category interaction $\left(\chi^{2}[1]=0.22, p=\right.$ .63) were significant (Figure 1A). 


\section{Delta-Plot Analyses}

A more complex pattern surfaces when examining the unfolding of the difference between taboo and non-taboo stimuli as a function of response speed. The reliable interference produced by Taboo connotation in fastest responses tended to level-off and show a reversal in the slowest ones (Figure 1B; see also Figure A1), irrespectively of stimulus lexical status. Consistently, there was a significant Quantile X Stimulus Category interaction $\left(\chi^{2}[4]=\right.$ 22.13, $p<.001)$, and no 3-way interaction $\left(\chi^{2}[4]=1.34, p=.25\right)$. The second order polynomial in fitting the variable Quantile significantly increased goodness-of-fit with respect to a model featuring solely a linear relationship $\left(\chi^{2}[2]=534.91, p<.001\right)$. Parameters for the final model are Reported in Table 2.

Table 2

Parameters of the final model for the analyses of EMG onsets as a function of response speed

\begin{tabular}{lcc}
\hline Random Effects & Variance & SD \\
\hline Participant & 9030 & 95.03 \\
Residual & 5304 & 72.83
\end{tabular}

\begin{tabular}{lccc} 
Fixed Effects & $\mathrm{b}$ & Std error & $\mathrm{t}$ \\
\hline Intercept & 790.83 & 15.14 & 52.22 \\
Lexicality (word) & -92.26 & 2.67 & $-34.60 *$ \\
Stimulus Cat. (Taboo) & 7.76 & 2.70 & $2.88 *$ \\
Quantile (linear) & 11720.35 & 102.76 & $114.06 *$ \\
Quantile (quadratic) & 1913.39 & 102.76 & $18.62 *$ \\
Lexicality (word) X Stimulus Cat (Taboo) & -8.63 & 3.78 & $-2.28 *$ \\
Stimulus Cat (Taboo) X Quantile (linear) & -714.91 & 145.67 & $-4.91 *$ \\
Stimulus Cat (Taboo) X Quantile & -406.22 & 145.67 & $-2.9 *$ \\
(quadratic) & & &
\end{tabular}

Note. Stimulus Cat. $=$ Stimulus Category (Taboo vs Non-Taboo). SD = standard deviation. Std error $=$ standard error. By-item random intercepts had to be dropped to prevent a singular fit. "Significant" coefficients $(\mathrm{t}>=|2|)$ are marked with an asterisk. 
In order to better investigate the interaction, follow-up models were conducted by fitting 2 separate models focusing, respectively, on the data from the first and fifth quantile. In these models, Stimulus Category and Lexicality were treated as fixed effects (in additive terms, as the interaction was not significant). Whereas the model focusing on the first quantile (i.e., the fastest RTs) displayed a significant interference from Taboo stimuli $\left(\chi^{2}[1]\right.$ $=14.44, p<.001 ; b=12.83, S E=3.35, t=3.83)$, in the last quantile the Taboo effect reverted to a significant facilitation $\left(\chi^{2}[1]=8.24, p=.004 ; b=-18.32, S E=6.38, t=-2.87\right)$.

\section{Sequential Analyses}

The reversal of the Taboo interference in the slowest quantile was interpreted as an index of suppression of interfering information via executive control functions. To further test this claim, a posteriori, we reasoned that this peculiar pattern may be emphasized when a Taboo item, as opposed to a Non-Taboo one, was displayed on the preceding trial. This prediction rests upon the notion that cognitive control can be enhanced via a top-down modulation after trials in which the interference was experienced (e.g., Gratton et al., 1992; Botvinick et al., 2001) and builds upon previous works showing the influence of the preceding trials in shaping the distributional profiles of conflict effects (e.g., Ridderinkhoff, 2002; Burle et al., 2002).

In this analysis, the Stimulus Category in item $\mathrm{N}-1$ was considered as an additional fixed effect. Before the analysis, we removed from the dataset a) the first response of each participant, and the first response after the break, b) all the responses for which an error or a partial error was made in the preceding trial (to avoid contamination from post-error slowing; Allain et al. 2009), and c) all the responses preceded by an invalid item. Quantiles were recomputed on this smaller subset of trials.

There was a significant 3-way interaction between Stimulus Category in trial $\mathrm{N}-1$, Stimulus Category in Trial N, and Quantile $\left(\chi^{2}[1]=9.63, p=.001\right)$. Fitting a second order 
polynomial for the variable Quantile significantly increased goodness-of-fit with respect to a model featuring a linear relationship $\left(\chi^{2}[2]=256.28, p<.001\right)$. Parameters of the full model are reported in Table 3.

Table 3

Parameters of the final model for the sequential analyses of EMG onset

\begin{tabular}{lcc}
\hline Random Effects & Variance & SD \\
\hline Participant & 9715.9 & 98.57 \\
Item & 278.10 & 16.68 \\
Residual & 4985.6 & 70.61
\end{tabular}

Fixed Effects

\begin{tabular}{ccc} 
b & Std error & $t$ \\
\hline 714.01 & 15.78 & 45.23
\end{tabular}

Stimulus Cat. (Taboo)

$19.90 \quad 3.52 \quad 5.65^{*}$

N-1 Stimulus Cat. (Taboo)

$17.74 \quad 3.05 \quad 5.81 *$

Quant. (linear)

$9538.03 \quad 147.77 \quad 64.54 *$

Quant. (quadratic)

$1225.13 \quad 144.25 \quad 8.49$ *

N-1 Stimulus Cat. (Taboo) X Stimulus

$\begin{array}{lll}-26.98 & 4.33 & -6.23 *\end{array}$

Cat. (Taboo)

Stimulus Cat. (Taboo) X Quant. (linear)

$\begin{array}{lll}111.25 & 208.92 & 0.53\end{array}$

Stimulus Cat. (Taboo) X Quant.

$\begin{array}{lll}-186.48 & 203.7 & -0.92\end{array}$

(quadratic)

N-1 Stimulus Cat. (Taboo) X Quant.

$437.02 \quad 203.34$

$2.15^{*}$

(linear)

N-1 Stimulus Cat. (Taboo) X Quant.

$146.22 \quad 202.30 \quad 0.72$
(quadratic)

N-1 Stimulus Cat. (Taboo) X Stimulus $\quad-934.39 \quad 287.72 \quad-3.25^{*}$

Cat. (Taboo) X Quant. (linear)

N-1 Stimulus Cat. (Taboo) X Stimulus $\quad-143.38 \quad 286.83 \quad-0.50$

Cat. (Taboo) X Quant. (quadratic)

Note. Stimulus Cat. = Stimulus Category (Taboo vs Non-Taboo). Quant. = Quantile. SD = standard deviation. Std error $=$ standard error. "Significant" coefficients $(\mathrm{t}>=|2|)$ are marked with an asterisk. 
After Non-Taboo stimuli, the interference exerted by the Taboo connotation seems to be reliable across most of the quantiles (Figure 1C; see also Figure A1). Follow-up analyses were conducted on trials following non-taboo stimuli, by fitting 5 separate models for each quantile, entering Stimulus Category as the fixed effect. The tests (using a false-discovery rate to control for multiple comparisons) revealed a significant Taboo interference in all quantiles (all $\chi^{2} \mathrm{~s}>6.06, p \mathrm{~s}<.018$; all $\left.b s>14.30, t \mathrm{~s}>2.48\right)$ except for the last one $\left(\chi^{2}[1]=\right.$ $2.39, p=.12 ; b=16.69, S E=10.75, t=1.55)$. In contrast, for trials following Taboo stimuli this interference is virtually non-existent, and turns into a facilitation effect for slower responses (Figure 1C; see also Figure A1). Follow-up models conducted within each quantile for trials following taboo stimuli, indeed, revealed no significant differences between taboo and non-taboo stimuli across the quantiles from 1 to 4 (all $\left.\chi^{2} \mathrm{~s}<4.11, p s>.10\right)$. A significant facilitation for taboo targets was instead detected in the fifth quantile $\left(\chi^{2}[1]=8.56, p=.017\right.$; $b=-31.23, S E=10.54, t=-2.96)$. 


\section{Figure 1}

Chronometric analyses of EMG onset.
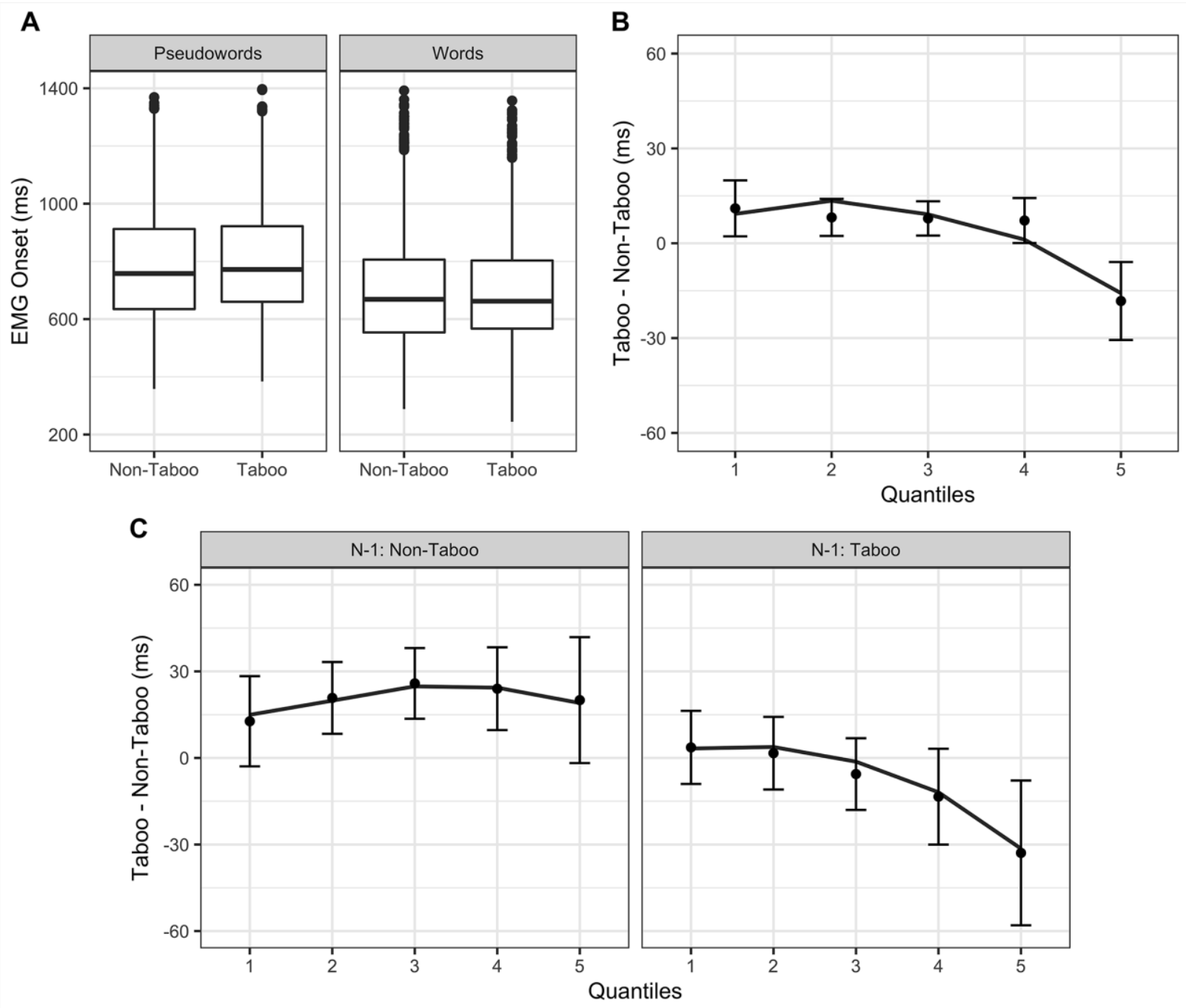

Note. A: EMG onset times (in ms) as a function of experimental conditions. B: Delta-plot: mean difference in EMG onset times between Taboo and Non-Taboo stimuli as a function of Quantiles (collapsed across stimulus lexicality). C: Sequential analyses. As in B, broken down as a function of Stimulus Category in the previous trial (N-1: Taboo vs N-1: NonTaboo). In B and C, points represent empirical means (error bars represent 95\% confidence intervals, adjusted for within-participants variables following Morey, 2008). Lines represent means predicted by the statistical model. 


\section{Partial Errors}

The analysis of partial errors was limited to trials with correct responses. There was a significant effect of Lexicality $\left(\chi^{2}[1]=13.72, p<.001\right)$, as partial errors were less likely to occur for words compared to pseudowords $(b=-0.39, S E=0.10, z=-3.80)$. There was no effect of Stimulus Category $\left(\chi^{2}[1]=0.00, p=.98\right)$. The interaction between the two factors was not significant $\left(\chi^{2}[1]=2.48, p=.11\right)$. Qualitatively, it seems that for pseudowords partial errors are more likely in the Taboo compared to the Non-Taboo conditions, whereas, if anything, the opposite is true for words (Figure $2 \mathrm{~A}){ }^{1}$

\section{Sequential analyses}

Despite the lack of any significant influence of taboo connotation on partial errors, we explored the possibility that a modulation may surface as a function of immediate trial history, due to adaptive changes in cognitive control. Specifically, we considered the fixed effects of Lexical Status on Trial N-1 and Stimulus Category on Trial N-1.Before the analyses, we trimmed the data following the same criteria specified for sequential analyses of EMG onsets. Results failed to highlight any effect of the factors coding for the status of the stimulus on trial $n-1$ either as main effects or in interaction with other factors $\chi^{2} \mathrm{~s}<3.74, p \mathrm{~s}$ >.053). However, when focusing on this more restricted selection of trials, the interaction between Lexicality and Stimulus Category reached conventional significance $\left(\chi^{2}[1]=5.36, p\right.$ $=.02$ ). The interaction seems to surface due to a higher chance of partial errors for taboo stimuli in case of pseudowords, whereas the opposite patterns seems to surface for words. Pairwise comparisons, however, failed to show any significant difference between non-taboo and taboo pseudowords $(b=-.25, S E=0.16, z=1.61, p=.11)$, or words $(b=.31, S E=0.17$, $z=1.75, p=.08)$ 


\section{Accuracy}

There was a significant effect of Lexicality $\left(\chi^{2}[1]=8.07, p=.004\right)$, with higher chance of accurate responses for words compared to pseudowords $(b=0.40, S E=0.14, z=2.911)$. Stimulus Category and the interaction between Lexicality and Stimulus Category failed to display significant effects $\left(\chi^{2} \mathrm{~s}<1, p \mathrm{~s}>.53\right)$. Proportion of correct responses as a function of conditions are represented in Figure 2B.

\section{Accuracy as a Function of Response Speed (Conditional Accuracy Functions)}

Despite the taboo connotation was not predictive of stimulus lexicality, there is the chance that the early availability of such a prepotent semantic feature may have biased participants towards "word" responses, yielding an inflated chance of lexicalization errors for taboo pseudowords, particularly in fast responses (i.e., lexical capture). To investigate this possibility, we explored how accuracy varies as a function of response times.

Trials in which participants did not provide a response and trials with inaccurate EMG onsets were removed from this analysis. Quantiles were computed on the remaining trials. There was a significant Lexicality by Quantile interaction $\left(\chi^{2}[1]=29.96, p<.001\right)$. The interaction clearly stems from an increased chance of fast errors for pseudowords compared to words (Figure 2B, right), pointing towards the occurrence of lexical captures. However, the phenomenon does not seem to be modulated by the Taboo connotation of the stimuli, as the 3-way interaction was not significant $\left(\chi^{2}[1]=0.63, p=.43\right)$. Further, there was no interaction between Quantile and Stimulus Category $\left(\chi^{2}[1]=0.38, p=.53\right)$. Parameters of the final model are reported in Table 4. 


\section{Table 4}

Parameters of the final model for the analysis of accuracy as a function of response speed (conditional accuracy functions)

\begin{tabular}{lcc}
\hline Random Effects & Variance & SD \\
\hline Participant & 0.42 & 0.65 \\
Item & 1.22 & 1.10
\end{tabular}

\begin{tabular}{lccc} 
Fixed Effects & $\mathrm{b}$ & Std error & $\mathrm{z}$ \\
\hline Intercept & 2.21 & 0.23 & 9.70 \\
Lexicality (Word) & 1.50 & 0.30 & $4.85^{*}$ \\
Quant. (linear) & 0.38 & 0.06 & $5.98^{*}$ \\
Lexicality (word) X Quant. (linear) & -0.48 & 0.09 & $-5.47^{*}$
\end{tabular}

Note. Quant. = Quantile. SD = standard deviation. Std error = standard error. "Significant" coefficients $(\mathrm{t}>=|2|)$ are marked with an asterisk.

\section{Figure 2}

Partial errors and response accuracy


Note. A: Proportion of partial errors as a function of experimental conditions. B: Proportion of correct responses as a function of experimental conditions (left); Conditional accuracy functions with proportion of accurate responses as a function of Lexicality (Pseudowords vs Words; collapsed across Stimulus Category) and Quantiles (right). Points represent empirical means (error bars represent 95\% confidence intervals, adjusted for within-participants variables following Morey, 2008). 


\section{General Discussion}

We investigated the role of cognitive control on semantic processing in visual word recognition by exploiting the detrimental effect of taboo information on lexical decision. Two different possibilities were assessed. First, cognitive control may intervene by dampening the taboo information via selective suppression mechanisms. This scenario was investigated by examining how the difference between taboo and non-taboo stimuli varies as a function of response speed, building on previous evidence in the field of cognitive control literature. Second, taboo connotation may enhance response monitoring, prompting a more careful consideration for taboo stimuli. This possibility was assessed by focusing on partial errors, i.e., trials in which an erroneous motor response is suppressed before completion. Results provided evidence for the selective suppression mechanisms and no reliable evidence of increased response monitoring for taboo stimuli in lexical decision.

Delta-plot analyses of RTs revealed that the interference exerted by the taboo connotation on lexical decision performance declines and actually reverses in the slowest responses. This pattern, rarely reported in visual word recognition, has been extensively documented in the context of the Simon effect, where it is interpreted as a marker of a selective suppression mechanism (e.g., Burle et al., 2002; Ridderinkhoff, 2002; for review, van den Wildenberg et al., 2010) that intervenes in order to dampen prepotent yet taskirrelevant information. We argue that the selective suppression we reported in the present experiment operates at the level of semantic information, rather than in relation to response conflict. As mentioned, the taboo connotation was not predictive of stimulus lexicality, and was thus not associated with any specific response option. Consistently, the reduction and reversal of taboo interference in slowest responses was not modulated by stimulus lexicality. The decline of taboo interference as a function of increasing RTs seems consistent with the 
notion of a suppression mechanism that operates on a distracting yet task-irrelevant semantic feature.

Converging evidence for an interpretation in terms of selective suppression mechanisms related to cognitive control came from sequential analysis considering immediate trial history (e.g., Ridderinkhoff, 2002). For trials following a taboo stimulus, taboo interference was virtually absent across the whole RTs distribution and showed a reversal in slower responses. Differently, a stable taboo interference surfaced after trials featuring neutral stimuli. This pattern is consistent with top-down adaptive modulations of flexible cognitive control mechanisms, which may be enhanced immediately after the presentation of interfering information (e.g., Botvinick et al., 2001; Stürmer et al., 2002).

Alternatively, a reduction of taboo interference in slower responses might indicate a passive decline of taboo interference: At the time in which slowest responses are delivered, taboo connotation might have exhausted its distracting influence. This interpretation is clearly at odds with respect to the adaptive modulation detected as a function of immediate trial history. Further, this passive decay account hardly explains the reversal of interference effect observed in the slowest quantile of RTs (Pratte et al., 2010). The reversal is more consistent with a suppression of the interfering semantic information, which may in turn increase the weighing in favor of task-relevant lexical-orthographic information and thus improve the performance.

Within extant models of visual word recognition, this processing dynamic could be captured by an adjustment of the relative contribution of orthographic and semantic processing to lexical decision. In models positing distributed representations for words across orthographic, phonological, and semantic representational levels (e.g., Harm \& Seidenberg, 2004; Plaut et al., 1996), readers might perform the lexical decision by, e.g., increasing reliance on orthographic units, rather than semantic ones. Differently, within computational 
models assuming localized representations, such as the DRC (Coltheart et al., 2001) and the CDP++ (Perry et al., 2010), this reasoning is complicated by the absence of semantic representational and processing levels within this framework. Instead, and in keeping with previous seminal works in the field (e.g., Balota, 1990; Balota et al., 1991; Balota \& Yap, 2006), our results suggest that semantic processing represents an integral part of visual word recognition, rather than an epiphenomenal, ballistic consequence of the "magic moment" of word recognition within the orthographic lexicon. This perspective clearly resonates with previous suggestions pointing towards the inclusion of a semantic layer within classic interactive activation model of word recognition (e.g., Balota et al., 1991). In this context, the early availability of semantic information granted by the cascaded flow of information across different representational and processing stages (e.g., Coltheart et al., 2001; Perry et al., 2010) would enable semantic activation to play a role in word recognition processes. In order to fully account for our results, this perspective may further need to endorse the notion of a flexible lexical processor (Balota \& Yap, 2006) that adaptively weighs the emphasis on different information codes (e.g., orthographic, semantic, phonological) as a function of task constraints and demands, in our case by dampening its reliance on prepotent yet taskirrelevant semantic information.

Differently, our experiment only provided weak evidence for enhanced responsemonitoring of taboo stimuli in lexical decision. Partial errors were more likely to occur for pseudowords than words, suggesting that response-monitoring might intervene to stop an impulsive word-response before it is finalized. Taboo connotation modulated this pattern only when focusing on the more restricted subset of trials considered for sequential analyses. Here, the Lexicality by Taboo Connotation interaction might point towards an increased chance of partial errors for taboo pseudowords. Speculatively, the result may indicate enhanced response-monitoring processes implemented to counteract a bias towards impulsive word- 
responses, prompted by the early availability of an additional semantic feature such as taboo features. However, the analysis of accuracy as a function of response speed (conditional accuracy functions) merely showed an increased chance of fast errors for pseudowords, without any additional modulation from Taboo Connotation. Thus, we found no evidence that fast and impulsive word-responses to word-like pseudowords are affected by the presence of a prepotent semantic feature such as taboo connotation.

However, despite these results, it would seem premature to completely dismiss any role of response-monitoring processes when facing taboo stimuli in visual word recognition. In fact, we focused on peripheral indexes of response monitoring, such as the correction of subthreshold motor response activation. It remains to be investigated whether earlier central indexes, such as response-related electrophysiological indexes of monitoring (e.g., Gratton et al., 2018) may highlight different outcomes, via finer-grained scrutiny of the dynamics of response selection and implementation.

We finally need to acknowledge one important limitation of our study, that is its inability to tease apart the unique contribution of taboo connotation over and above emotional valence (and arousal). Previous evidence suggests that the processing of taboo words is determined by specific factors and properties (e.g., tabooness), over and above the influence of valence and arousal (e.g., Madan et al., 2017). It is indeed worth emphasizing that whereas taboo words have been consistently associated to interference effects (e.g., Carretié et al., 2008; Madan et al., 2017; Sulpizio et al., 2019; 2020), negative emotive words have been associated to both detrimental (e.g., Estes \& Adelman, 2008) and facilitatory effects (e.g., Kousta et al., 2009). This discrepancy seems to further point towards a unique status of taboo words. Indeed, our reliance on this specific class of words was motivated by their ability to consistently trigger a semantic interference, which may in turn call for the intervention of executive control mechanisms to dampen detrimental effects on the achievements of the 
task's goal. Ultimately, the more general overlap with emotive words remains to be assessed within specific experiments, possibly implementing a direct comparison across these different types of stimuli (taboo words, positive and negative emotive words).

In conclusion, the present experiment shows evidence that, during visual word recognition, cognitive control mechanisms may intervene to regulate the activation of prepotent yet task-irrelevant semantic information. Specifically, selective suppression mechanisms may counteract the detrimental effect of taboo connotation by dampening its influence and restoring a reliance on task-relevant information. This perspective is consistent with recent proposals (Lambon-Ralph et al., 2017) suggesting that semantic processing crucially hinges upon control mechanisms that support a goal-oriented weighing of semantic information. Top-down control processes might use the task's goal to bias activation within the system and direct it toward task-appropriate behaviors while suppressing task-irrelevant information.

In endorsing this perspective, we argue that our results may provide important insight into two related issues. The influence of semantic variables on visual word recognition has been highlighted in other previous works (see Pexman, 2012, for a review). Here, we further provide evidence of an adaptive controlled processing of semantic information in the context of a pure recognition task. In particular, our results highlight the intervention of cognitive control on semantic processing via a selective suppression mechanism that dampens the influence of prepotent yet task-irrelevant information. Notably, compared to semantic classification and/or decision tasks typically used to study conceptual processing and semantic control (e.g., Chiou et al., 2018; Montefinese et al., 2020), the lexical decision task does not explicitly require the online weighing of semantic features (e.g., concrete vs. abstract, living vs. non-living; e.g., Kiefer et al., 2008). Nonetheless, dynamics of semantic control appear to be critically involved in lexical decision as well, highlighting a potential 
common ground between models of visual word recognition and recent models of conceptual processing (e.g., Hoffman et al., 2018). Second, and relatedly, our results suggest a reconsideration of the role of semantic processing (and control) in visual word recognition. Rather than an epiphenomenal consequence of word recognition, semantic processing, in its interaction with control mechanisms, may actually represent a flexible and goal-directed keycomponent of visual word recognition (e.g., Balota et al., 1991; Balota \& Yap, 2006), on par with orthographic decoding and lexical access.

Open Practices and Data Availability Statements. Data and supplementary materials for the experiment reported here are available at https://osf.io/jq8kv/. The experiment was not preregistered. 


\section{References}

Allain, S., Burle, B., Hasbroucq, T., \& Vidal, F. (2009). Sequential adjustments before and after partial errors. Psychonomic Bulletin \& Review, 16, 356-362.

Amenta, S., Marelli, M., \& Sulpizio, S. (2017). From sound to meaning: Phonology-tosemantics mapping in visual word recognition. Psychonomic bulletin \& review, 24, 887-893.

Aschenbrenner, A. J., Balota, D. A., Weigand, A. J., Scaltritti, M., \& Besner, D. (2017). The first letter position effect in visual word recognition: The role of spatial attention. Journal of Experimental Psychology: Human Perception and Performance, 43, 700718.

Bates, D., Maechler, M., Bolker, B., Walker, S., Christensen, R. H. B., Singmann, H., ... \& Bolker, M. B. (2015). Package 'lme4'. Convergence, 12, 2.

Balota, D. A. (1990). The role of meaning in word recognition. In D. A. Balota, G. B.Flores d'Arcais, \& K. Rayner (Eds.), Comprehension processes in reading (pp. 9-32). Hillsdale, NJ: Lawrence Erlbaum Associates, Inc.

Balota, D. A., Ferraro, F. R., \& Connor, L. T. (1991). On the early influence of meaning in word recognition: A review of the literature. In P. J. Schwanenflugel (Ed.), The psychology of word meanings (pp.187-222). Hillsdale, NJ: Erlbaum.

Balota, D. A., \& Yap, M. J. (2006). Attentional control and the flexible lexical processor: Explorations of the magic moment of word recognition. From inkmarks to ideas: Current issues in lexical processing. In S. Andrews (Ed.), From inkmarks to ideas: Current issues in lexical processing (pp. 229-258). New York, NY: Psychology Press.

Besner, D., Risko, E.F., Stolz, J.A., White, D., Reynolds, M., O’Malley, S., \& Robidoux, S. (2016). Varieties of attention: Their roles in visual word identification. Current Directions in Psychological Sciences, 25, 162-168. 
Botvinick, M., \& Braver, T. (2015). Motivation and cognitive control: from behavior to neural mechanism. Annual review of psychology, 66, 83-113.

Botvinick, M. M., Braver, T. S., Barch, D. M., Carter, C. S., \& Cohen, J. D. (2001). Conflict monitoring and cognitive control. Psychological review, 108, 624-652.

Bradley, M. M., \& Lang, P. J. (1999). Affective norms for English words (ANEW): Instruction manual and affective ratings (Vol. 30, No. 1, pp. 25-36). Technical report C-1, the center for research in psychophysiology, University of Florida.

Burle, B., Possamaï, C. A., Vidal, F., Bonnet, M., \& Hasbroucq, T. (2002). Executive control in the Simon effect: an electromyographic and distributional analysis. Psychological Research, 66, 324-336.

Carretié, L., Hinojosa, J. A., Albert, J., López-Martín, S., De La Gándara, B. S., Igoa, J. M., \& Sotillo, M. (2008). Modulation of ongoing cognitive processes by emotionally intense words. Psychophysiology, 45, 188-196.

Cassani, G., Chuang, Y.-Y., \& Baayen, R. H. (2020). On the semantics of nonwords and their lexical category. Journal of Experimental Psychology: Learning, Memory, and Cognition, 46, 621-637.

Chiou, R., Humphreys, G. F., Jung, J., \& Lambon Ralph, M. A. (2018). Controlled semantic cognition relies upon dynamic and flexible interactions between the executive 'semantic control' and hub-and-spoke 'semantic representation' systems. Cortex, 103, 100-116.

Chuang, Y. Y., Vollmer, M. L., Shafaei-Bajestan, E., Gahl, S., Hendrix, P., \& Baayen, R. H. (2020). The processing of pseudoword form and meaning in production and comprehension: A computational modeling approach using Linear Discriminative Learning. Behavior Research Methods. Advanced online publication. https://doi.org/10.3758/s13428-020-01356-w. 
Coltheart, M., Rastle, K., Perry, C., Langdon, R., \& Ziegler, J. (2001). DRC: a dual route cascaded model of visual word recognition and reading aloud. Psychological Review, 108, 204-256.

Crepaldi, D., Keuleers, E., Mandera, P., \& Brysbaert, M. (2013). SUBTLEX-IT. Retrieved from http://crr.ugent.be/subtlex-it/

de Jong, R., Liang, C.-C., \& Lauber, E. (1994). Conditional and unconditional automaticity: A dual process model of effects of spatial stimulus-response correspondence. Journal of Experimental Psychology: Human Perception and Performance, 20, 731-750.

Dhooge, E., \& Hartsuiker, R. J. (2011). How do speakers resist distraction? Evidence from a taboo picture-word interference task. Psychological Science, 22, 855-859.

Estes, Z., \& Adelman, J. S. (2008). Automatic vigilance for negative words in lexical decision and naming: Comment on Larsen, Mercer, and Balota (2006). Emotion, 8, 441444.

Gratton, G., Coles, M. G., \& Donchin, E. (1992). Optimizing the use of information: strategic control of activation of responses. Journal of Experimental Psychology: General, 121, 480-506.

Gratton, G., Cooper, P., Fabiani, M., Carter, C. S., \& Karayanidis, F. (2018). Dynamics of cognitive control: Theoretical bases, paradigms, and a view for the future. Psychophysiology, 55, e13016.

Hansen, S. J., McMahon, K. L., Burt, J. S., \& de Zubicaray, G. I. (2017). The locus of taboo context effects in picture naming. The Quarterly Journal of Experimental Psychology, 70, $75-91$. 
Harm, M. W., \& Seidenberg, M. S. (2004). Computing the meanings of words in reading: cooperative division of labor between visual and phonological processes. Psychological review, 111, 662-720.

Hendrix, P., \& Sun, C. C. (2020). A word or two about nonwords: Frequency, semantic neighborhood density, and orthography-to-semantics consistency effects for nonwords in the lexical decision task. Journal of Experimental Psychology: Learning, Memory, and Cognition. Advanced online publication. https://doi.org/10.1037/xlm000

Hoffman, P., McClelland, J. L., \& Lambon Ralph, M. A. (2018). Concepts, control, and context: A connectionist account of normal and disordered semantic cognition. Psychological Review, 125, 293-328.

Kiefer, M., Sim, E. J., Herrnberger, B., Grothe, J., \& Hoenig, K. (2008). The sound of concepts: four markers for a link between auditory and conceptual brain systems. Journal of Neuroscience, 28, 12224-12230.

Kousta, S. T., Vinson, D. P., \& Vigliocco, G. (2009). Emotion words, regardless of polarity, have a processing advantage over neutral words. Cognition, 112, 473-481.

Lambon Ralph, M. A., Jefferies, E., Patterson, K., \& Rogers, T. T. (2017). The neural and computational bases of semantic cognition. Nature Reviews Neuroscience, 18, 42-55.

MacKay, D. G., Shafto, M., Taylor, J. K., Marian, D. E., Abrams, L., \& Dyer, J. R. (2004). Relations between emotion, memory, and attention: Evidence from taboo Stroop, lexical decision, and immediate memory tasks. Memory \& Cognition, 32, 474-488.

Madan, C. R., Shafer, A. T., Chan, M., \& Singhal, A. (2017). Shock and awe: Distinct effects of taboo words on lexical decision and free recall. Quarterly Journal of Experimental Psychology, 70, 793-810. 
Mädebach, A., Markuske, A. M., \& Jescheniak, J. D. (2018). When does reading dirty words impede picture processing? Taboo interference with verbal and manual responses. Psychonomic Bulletin \& Review, 25, 2301-2308.

Marelli, M., \& Amenta, S. (2018). A database of orthography-semantics consistency (OSC) estimates for 15,017 English words. Behavior research methods, 50, 1482-1495.

McCann, R. S., Folk, C. L., \& Johnson, J. C. (1992). The role of spatial attention in visual word processing. Journal of Experimental Psychology: Human Perception and Performance, 18, 1015-1029.

Montefinese, M., Ambrosini, E., Fairfield, B., \& Mammarella, N. (2014). The adaptation of the affective norms for English words (ANEW) for Italian. Behavior Research Methods, 46, 887-903.

Montefinese, M., Hallam, G., Thompson, H. E., \& Jefferies, E. (2020). The interplay between control processes and feature relevance: Evidence from dual-task methodology. Quarterly Journal of Experimental Psychology, 73, 384-395.

Morey, R. D. (2008). Confidence intervals for normalized data. A correction to Cousineau (2005). Tutorials in Quantitative Methods for Psychology, 4, 61-64.

Motley, M. T., Camden, C. T., \& Baars, B. J. (1981). Toward verifying the assumptions of laboratory-induced slips of the tongue: The output-error and editing issues. Human Communication Research, 8, 3-15.

Motley, M. T., Camden, C. T., \& Baars, B. J. (1982). Covert formulation and editing of anomalies in speech production: Evidence from experimentally elicited slips of the tongue. Journal of Verbal Learning and Verbal Behavior, 21, 578-594. 
O'Malley, S., \& Besner, D. (2008). Reading aloud: Qualitative differences in the relation between stimulus quality and word frequency as a function of context. Journal of Experimental Psychology: Learning, Memory, and Cognition, 34, 1400-1411.

Perry, C., Ziegler, J. C., \& Zorzi, M. (2010). Beyond single syllables: Large-scale modeling of reading aloud with the Connectionist Dual Process (CDP++) model. Cognitive psychology, 61, 106-151.

Pexman, P. M. (2012). Meaning-based influences on visual word recognition. In J. S. Adelman (Ed.), Visual word recognition: Volume 2 (pp. 24-43). New York, NY: Psychology Press.

Plaut, D. C., McClelland, J. L., Seidenberg, M. S., \& Patterson, K. (1996). Understanding normal and impaired word reading: computational principles in quasi-regular domains. Psychological review, 103, 56-115.

Pratte, M. S., Rounder, J. N., Morey, R. D., \& Feng, C. (2010). Exploring the differences in distributional properties between Stroop and Simon effects using delta plots. Attention, Perception, \& Psychophysics, 72, 2013-2025.

R Core Team (2015). R: A language and environment for statistical computing. Vienna, Austria: R Foundation for Statistical Computing. http://www.R-project.org/.

Ridderinkhof, K. R. (2002). Micro- and macro-adjustments of task set: activation and suppression in conflict tasks. Psychological Research, 66, 312-323.

Siegrist, M. (1995). Effects of taboo words on color-naming performance on a Stroop test. Perceptual and Motor Skills, 81, 1119-1122. 
Scaltritti, M., Balota, D. A., \& Peressotti, F. (2013). Exploring the additive effects of stimulus quality and word frequency: The influence of local and list-wide prime relatedness. The Quarterly Journal of Experimental Psychology, 66, 91-107.

Stürmer, B., Leuthold, H., Soetens, E., Schröter, H., \& Sommer, W. (2002). Control over location-based response activation in the Simon task: behavioral and electrophysiological evidence. Journal of Experimental Psychology: Human Perception and Performance, 28, $1345-1363$.

Sulpizio, S., Pennucci, E., \& Job, R. (2020). The impact of emotional content on pseudoword recognition. Psychological Reserch. https://doi.org/10.1007/s00426-020-01454-6

Sulpizio, S., Toti, M., Del Maschio, N., Costa, A., Fedeli, D., Job, R., \& Abutalebi, J. (2019). Are you really cursing? Neural processing of taboo words in native and foreign language. Brain and language, 194, 84-92.

Sulpizio, S., Vassallo, E., Job, R., \& Abutalebi, J. (2020). ITABÙ: Dati preliminari di un database delle parole tabù per l'italiano [ITABOO: Preliminary data for an Italian database for taboo words]. Giornale Italiano di Psicologia, 2, 599-614.

Van Den Wildenberg, W. P., Wylie, S. A., Forstmann, B. U., Burle, B., Hasbroucq, T., \& Ridderinkhof, K. R. (2010). To head or to heed? Beyond the surface of selective action inhibition: a review. Frontiers in Human Neuroscience, 4, 222.

White, K. K., Abrams, L., Koehler, S. M., \& Collins, R. J. (2017). Lions, tigers, and bears, oh sh! t: Semantics versus tabooness in speech production. Psychonomic bulletin \& review, $24,489-495$.

White, K. K., Abrams, L., LaBat, L. R., \& Rhynes, A. M. (2016). Competing influences of emotion and phonology during picture-word interference. Language, Cognition and Neuroscience, 31, 265-283. 
Yap, M. J., Balota, D. A., Tse, C. S., \& Besner, D. (2008). On the additive effects of stimulus quality and word frequency in lexical decision: evidence for opposing interactive influences revealed by RT distributional analyses. Journal of Experimental Psychology: Learning, Memory, and Cognition, 34, 495-513. 


\section{Footnotes}

1. Partial errors reflect the suppressed activation of the incorrect responses. In the context of the Simon task, a steeper reduction of the Simon effect for the slowest responses has been observed for trials containing partial EMG errors, reflecting the strong intervention of suppression mechanisms (Burle et al., 2002). Unfortunately, the exploration of this issue in the current experiment was prevented by the fact that for many participants partial errors were too few to reliably assess partial errors as a function of response speed. 


\section{Appendix}

\section{Quantile Plots}

Figure A1. Quantile plots showing mean EMG onset time as a function of experimental conditions and quantiles.

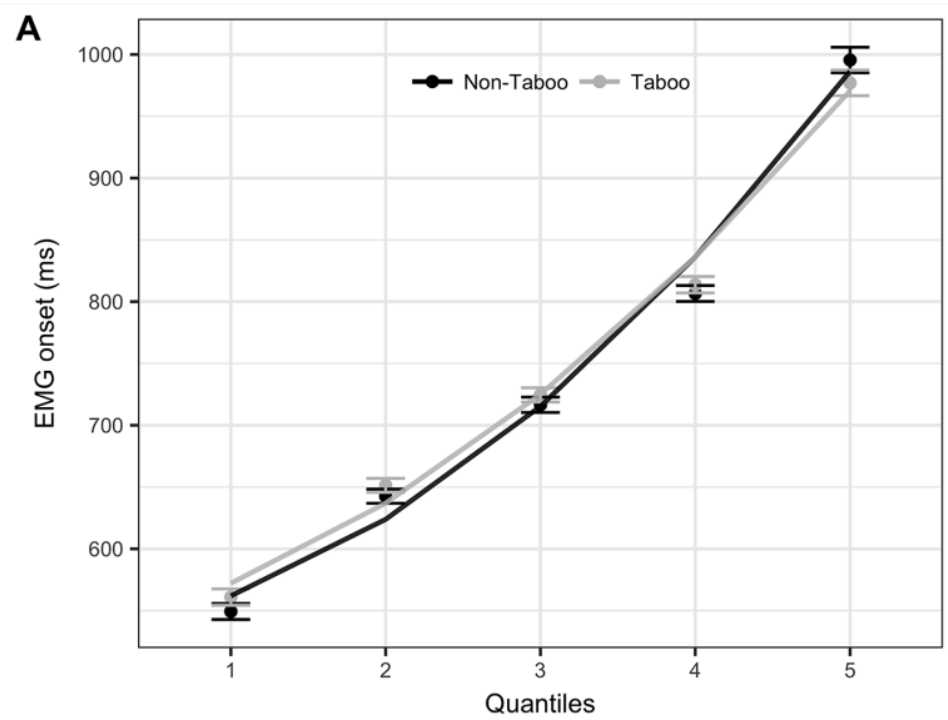

B

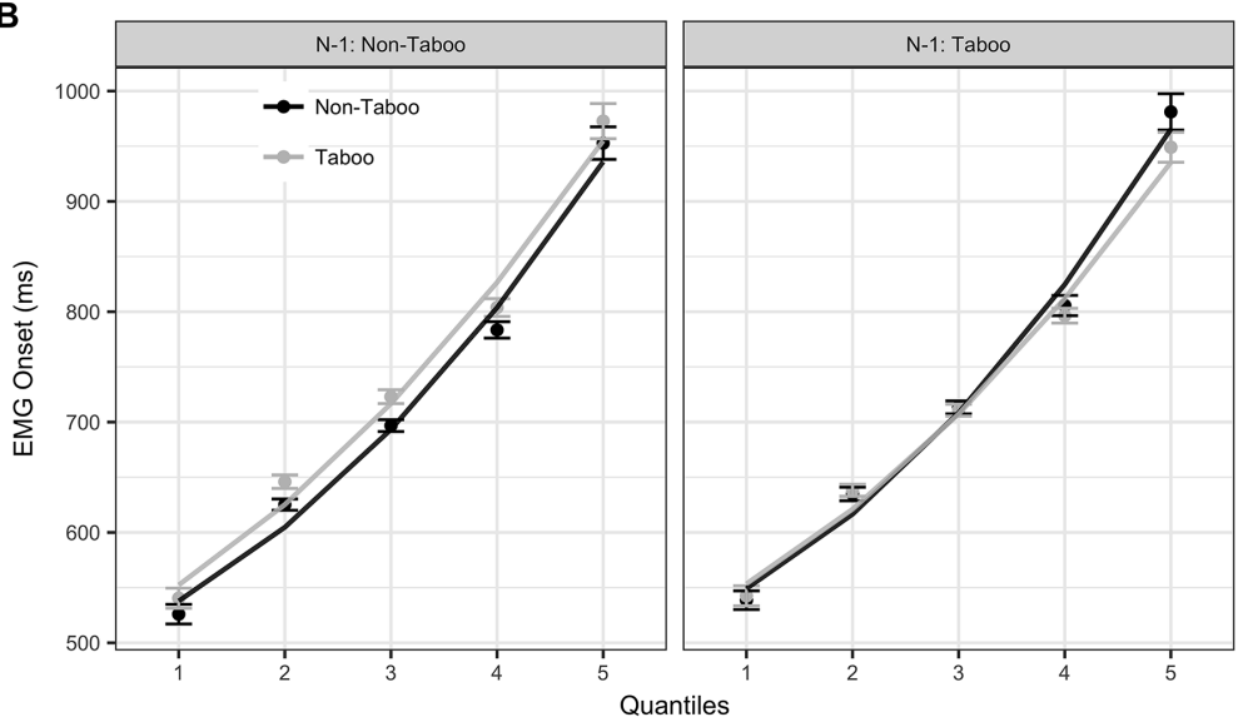

Note. A: EMG onset times as a function of Stimulus Category (Taboo vs Non-Taboo) and Quantiles (collapsed across stimulus lexicality). B: As in A, broken down as a function of Stimulus Category in the previous trial (N-1: Taboo vs N-1: Non-Taboo). Points represent empirical means (error bars represent 95\% confidence intervals, adjusted for withinparticipants variables following Morey, 2008). Lines represent means predicted by the statistical model. 